\title{
THE APRIL MEETING IN NEW YORK
}

The four hundred sixty-eighth meeting of the American Mathematical Society was held at Columbia University, New York City, on Friday and Saturday, April 27-28, 1951. The meeting was attended by about 250 persons, including the following 217 members of the Society:

C. R. Adams, E. J. Akutowicz, R. D. Anderson, R. L. Anderson, T. W. Anderson, R. G. Archibald, Helmut Aulbach, Maurice Auslander, M. C. Ayer, Joshua Barlaz, L. W. Baumhoff, E. G. Begle, A. A. Bennett, Stefan Bergman, Lipman Bers, Nicholas Bilotta, D. W. Blackett, E. K. Blum, Raoul Bott, Samuel Bourne, A. T. Brauer, Paul Brock, F. E. Browder, A. B. Brown, R. G. Brown, R. H. Brown, M. L. Browne, L. J. Burton, J. H. Bushey, Jewell H. Bushey, F. P. Callahan, W. R. Callahan, R. R. Christian, K. L. Chung, F. E. Clark, A. B. Clarke, F. M. Clarke, Eckford Cohen, L. W. Cohen, R. M. Cohn, T. F. Cope, Natalie Coplan, H. S. M. Coxeter, J. B. Crabtree, C. W. Curtis, P. H. Daus, J. C. E. Dekker, J. B. Diaz, J. A. Dieudonné, Jesse Douglas, Nelson Dunford, William H. Durfee, Aryeh Dvoretzky, B. M. Dwork, J. S. Dwork, M. E. Estill, R. M. Exner, W. H. Fagerstrom, Herbert Federer, J. M. Feld, William Feller, Werner Fenchel, D. T. Finkbeiner, R. S. Finn, Irwin Fischer, H. A. Forrester, R. M. Foster, J. S. Frame, Gerald Freilich, F. N. Frenkiel, Bernard Friedman, K. O. Friedrichs, R. F. Gabriel, G. N. Garrison, H. M. Gehman, W. M. Gilbert, B. P. Gill, Leonard Gillman, Sidney Glusman, H. E. Goheen, J. K. Goldhaber, Oscar Goldman, R. D. Gordon, Daniel Gorenstein, J. A. Greenwood, Laura Guggenbuhl, Carl Hammer, P. C. Hammer, G. A. Hedlund, M. H. Heins, Alex Heller, Aaron Herschfeld, E. H. C. Hildebrandt, T. H. Hildebrandt, Einar Hille, A. J. Hoffman, S. P. Hoffman, Banesh Hoffmann, L. A. Hostinsky, E. M. Hull, T. R. Humphreys, L. C. Hutchinson, B. M. Ingersoll, Nathan Jacobson, R. E. Johnson, R. V. Kadison, Shizuo Kakutani, Irving Kaplansky, Edward Kasner, M. E. Kellar, J. F. Kiefer, H. S. Kieval, J. R. Kline, E. R. Kolchin, B. O. Koopman, Saul Kravetz, Helen Kutman, M. K. Landers, V. V. Latshaw, Solomon Lefschetz, Benjamin Lepson, Howard Levene, M. E. Levenson, W. G. Lister, L. H. Loomis, E. R. Lorch, Eugene Lukacs, N. H. McCoy, L. A. MacColl, G. W. Mackey, H. M. MacNeille, Wilhelm Magnus, Irwin Mann, Murray Mannos, W. T. Martin, F. I. Mautner, A. E. Meder, Jr., A. N. Milgram, K. S. Miller, W. H. Mills, M. L. Minsky, Don Mittleman, F. J. Murray, W. R. Murray, John von Neumann, C. V. Newsom, A. V. Newton, O. M. Nikodým, P. B. Norman, A. F. O'Neill, J. C. Oxtoby, G. W. Patterson, L. E. Payne, Anna Pell-Wheeler, R. S. Phillips, G. B. Price, M. H. Protter, Hans Rademacher, G. N. Raney, H. E. Rauch, H. W. Reddick, Helene Reschovsky, Moses Richardson, C. E. Rickart, R. E. Roberson, J. H. Roberts, H. P. Robertson, M. S. Robertson, G. B. Robison, I. H. Rose, Maxwell Rosenlicht, H. D. Ruderman, James Sanders, Arthur Sard, L. R. Sario, S. W. Saunders, I. R. Savage, R. D. Schafer, E. C. Schlesinger, Abraham Schwartz, C. H. W. Sedgewick, M. A. Shader, Dorothy Shaffer, James Singer, P. A. Smith, Andrew Sobczyk, J. J. Sopka, Fritz Steinhardt, R. W. Stokes, D. M. Stone, Walter Strodt, M. M. Sullivan, Olga Taussky, E. W. Titt, P. M. Treuenfels, A. W. Tucker, J. W. Tukey, J. R. Van Andel, H. E. Vansant, D. F. Votaw, R. J. Walker, J. L. Walsh, W. G. Warnock, Alan Wayne, J. V. Wehausen, Alexander Weinstein, Morris Weisfeld, Louis Weisner, J. G. Wendel, Franc Wertheimer, G. N. White, G. T. Whyburn, M. A. Woodbury, Arthur Wouk, L. A. Zadeh, Oscar Zariski, Arthur Zeichner. 
At a general session on Friday Professor Herbert Federer of Brown University delivered an invited address on Measure and area, Professor G. B. Price presiding. An invited address on $\sigma$-complete Boolean algebras was delivered by Professor L. H. Loomis of Harvard University at a general session on Saturday, Professor E. R. Kolchin presiding.

The session for contributed papers on Friday was presided over by Professor R. S. Phillips. Professor J. S. Frame and Professor J. H. Roberts presided at the Saturday morning sessions.

The Council met at the Faculty Club at 8:00 P.M. on April 27.

The Secretary announced the election of the following forty-five persons to ordinary membership in the Society:

Assistant Professor Joseph Andrushkiw, Seton Hall University;

Mr. Lester Glenn Arnold, Teaching Assistant, University of Rochester;

Mr. Lester W. Baumhoff, Staff Member, Los Alamos Scientific Laboratory;

Mr. Aaron Besner, Physicist, Norden Laboratories Corporation, White Plains, N.Y.;

Mr. David Alvin Buchsbaum, Lecturer, Columbia University;

Mr. Francis Patrick Callahan, Jr., Mathematician and Physicist, Research Laboratories, Interchemical Corporation, New York, N.Y.;

Mr. Rene Felix Dennemeyer, Research Engineer, North American Aviation Company, Aerophysics Laboratory, Downey, Calif.;

Mr. James D. Esary, Teaching Assistant, University of California, Berkeley 4, Calif.;

Mr. Louis Samuel Gage, Instructor, Radar Department, Keesler Air Force Base, Biloxi, Miss.;

Mr. Deba Brata Ghosh, Assistant, Reserve Bank of India, Calcutta, India;

Mr. Raef K. Haddad, Teaching Assistant, Psychology Department, New York University;

Mr. Bradford Frank Hadnot, Instructor, North Georgia College, Dahlonega, Ga.;

Mr. Lawrence Harris, Research Engineer, Norden Laboratories Corporation, White Plains, N.Y.;

Mr. Joseph Owens Harrison, Jr., Assistant Supervisor, Computational Analysis Laboratory, Eckert-Mauchly Computer Corporation, Philadelphia, Pa.;

Mr. Herbert Milton Heughan, Instructor, Agricultural and Technical College, Greensboro, N.C.

Miss Gladys Naxiene Hurt, Instructor, Morehouse College;

Mr. Shikao Ikehara, Staff Member, Research Laboratory of Electronics, Massachusetts Institute of Technology;

Dr. Salem Hanna Khamis, Statistician, Statistical Office, United Nations, New York, N.Y.;

Mr. V. V. Lavroff, Comptroller, Atlanta Division, University of Georgia, Atlanta, Ga.;

Sister Catherine Marie Lee, Instructor, Trinity College, Washington, D.C.;

Miss Louise L. Leon, Actuarial Department, Guardian Life Insurance Company, New York, N.Y.;

Sister Mary Ferrer McFarland, Instructor, St. Xavier College, Chicago 15, Ill.;

Mr. John Patterson Mayberry, Research Assistant, Princeton University;

Mr. Henry I. Meyer, Mathematician, United Gas Corp., Shreveport, La. 
Associate Professor Robbie Lee Beaven Morris (Mrs. Andrew F.), Cumberland University, Lebanon, Tenn.;

Associate Professor Harry Ernest Nelson, Augustana College, Rock Island, Ill.;

Mr. Frederick Esser Nemmers, Milwaukee, Wis.;

Assistant Professor Charles Jefferson Pipes, Jr., Southern Methodist University;

Mr. Harold L. Platzer, Columbia University;

Mr. Solomon Leonard Pollack, Statistical Draftsman, Office of Surgeon General, Washington, D.C.;

Mr. John Francis Ries, Instructor, Kings College, Kings College, Del.;

Mr. George Schaeffer Ritchie, University of Michigan;

Mr. Paul George Rooney, Assistant, California Institute of Technology;

Dr. Edward Walter Samson, Chief, Communications Laboratory, Air Force Cambridge Research Laboratories, Cambridge, Mass.;

Mr. Earl Justin Schweppe, University of Illinois;

Mr. Raymond Sedney, Teaching Assistant, Carnegie Institute of Technology;

Mr. Frederico Mariano Sioson, Pantihan No. 1, Naval Street, Malabon, Rizal, Philippines;

Dr. Roy C. Spencer, Chief, Antenna Laboratory, Air Force Cambridge Research Laboratories, Cambridge, Mass.;

Mr. Robert Delmer Stalley, Instructor, University of Arizona;

Mr. Carl Worth Steeg, Jr., Instructor, Massachusetts Institute of Technology;

Mr. Marvin Stern, Consultant, Republic Aviation Corporation, Farmingdale, N.Y.; Mr. Carl Henry Tross, Mathematician, U. S. Naval Proving Ground, Dahlgren, Va.; Mr. Michael Anthony Tschupp, Testing Engineer, Electric Specialty Company, South Street, Stamford, Conn.;

Mr. Richard Joseph Turyn, Harvard University;

Mr. Howard Raymond Wright, Jr., Aeronautical Engineer, Latin-American Division, Pan-American Airways, Miami, Fla.;

Mr. Herbert S. Wolf, Assistant, University of Illinois;

It was reported that the following seventy persons had been elected as nominees of institutional members as indicated:

University of Alabama: Mr. Thomas Curtis Yarbrough.

University of British Columbia: Mr. Ronald Alvin Macauley.

California Institute of Technology: Messrs. Douglas Robert Anderson and Richard Scott Pierce.

University of Chicago: Messrs. George Edward Backus, William Ralph Ballard, Joseph Bram, Arlen H. Brown, Ernest Lyle Griffin, Jr., Frank Douglas Quigley, Andrew Hugh Wallace, and Oswald Wyler.

University of Cincinnati: Messrs. Hugo Heermann and Frank Levin.

City College of New York: Messrs. Barry Bernstein, Jacob Leon Bricker, Herman Hanisch, and Harold Widom.

Columbia University: Messrs. Paul Otokar Backer, Enrique Bayo, Roberto Joseph Beeber, Lawrence Goldman, and Katsumi Nomizu.

Cornell University: Messrs. John Wells Brace, Tseng Yeh Chow, and Jean-Pierre G. Meyer.

Harvard University: Messrs. Kurt Bing and Zdziskaw Alexander Melzak.

Institute for Advanced Study: Professor Rudolf Inzinger.

Kenyon College: Mr. Lane Schofield Hart, IV. 
Michigan State College: Messrs. John William Coy, Rubin Kuritsky, and Richard Zindler.

New York University: Messrs. Peter Ceike, Richard Goldberg, and Leon Weliczker. Northwestern University: Messrs. Thomas Cleveland Littlejohn and James Sparks Thale.

Oklahoma Agricultural and Mechanical College: Mr. Allan Kellerman Jennings.

State University of Iowa: Mr. Howard Henry Wicke.

Syracuse University: Dr. Jim Christoph Edmond Dekker, Miss Margaret A. Finn, and Mr. Melvin Aaron Shader.

University of Illinois: Mr. Thomas Blaney Elfe, Jr.

University of Michigan: Mr. Walter Ferdinand Bauer.

University of Minnesota: Mr. William O. S. Moser.

University of Missouri: Mr. Steven J. Bryant.

University of Texas: Messrs. David Fleming Dawson, Bennie J. Pearson, and John Marshall Slye.

University of Toronto: Messrs. Jean Eugene LeBel, Gordon Marshall Petersen, and Richard James Semple.

University of Virginia: Messrs. Julian Brody, Ralph Warren Jollensten, Wendell Endicott Malbon, and Robert Lee Plunkett.

University of Washington: Mr. Calvin Abraham Rogers.

University of Wisconsin: Messrs. Henry Beiman, Wilbur Eugene Deskins, Carl Edwin Diesen, Trevor Evans, Philip Wayne Gillett, Adrien Leroy Hess, Lucien Wolf Neustadt, Marshall Flynn Ruchte, Robert Louis San Soucie, and Bernard Witt. Vanderbilt University: Mr. Thomas Lee Jordan.

The Secretary announced that the following had been admitted to the Society in accordance with reciprocity agreements with various mathematical organizations: London Mathematical Society: $\mathrm{Mr}$. James Alexander Edmond Black, Research Laboratories, General Electric Company, Stanmore, Middlesex, England; Professor Jacob Lionel Bakst Cooper, University College, Cardiff, Wales; Société Mathématique de France: Professor André Jean-Marie Combes, Versailles Lycée, Mantes-Gassicourt, Seine at Oise, France; Professor Abolghassen Ghaffari, Teheran University, Teheran, Iran; Professor Sophie Piccard, University of Neuchâtel, Neuchâtel, Switzerland; Unione Matematica Italiana: Professor Francesco Cecioni, Institute of Mathematics, University of Pisa, Pisa, Italy; Dr. Carmelo Roberto Fusa, National Institute of Higher Mathematics, Rome, Italy; Wiskundig Genootschap te Amsterdam: Dr. Leonard Lips, Lecturer, University of Indonesia, Bandung, Java, Indonesia.

Queens College, New York, N. Y. was elected to institutional membership.

The following actions taken by mail vote of the Council were reported: election of Professors W. Feller and M. H. Heins as members of the Executive Committee of the Council for a period of two years beginning January, 1951; approval of the recommendation of the 
Policy Committee that adherence to the International Mathematical Union be through the National Research Council; the election of North Texas State Teachers College to institutional membership.

The following appointment of a representative of the Society was reported: Mary Ann Lee at inauguration of John Rutherford Everett as President of Hollins College on April 16, 1951.

The following additional appointments by the President were reported: T. F. Cope and B. P. Gill as auditors of the Society's accounts for 1951; E. Lukacs (Chairman), Leon Cohen, E. J. Cox, F. E. Johnston, O. J. Ramler, F. D. Rigby, and F. W. Cohon as a committee on arrangements for Washington, D. C. meeting October 27, 1951; A. E. Meder, Jr. (Chairman), H. M. MacNeille, and P. A. Smith as a committee to make recommendations on policy on bequests; C. R. Adams (Chairman), E. R. Lorch, and D. V. Widder as a committee to consider dues of members who are wives of members; J. R. Kline (Chairman), Oswald Veblen, and G. T. Whyburn as a committee for consultation on the Duke Mathematical Journal; G. A. Hedlund (Chairman), William Feller, M. R. Hestenes, F. B. Jones, and S. B. Myers as a committee to nominate officers and members of the Council for 1952; A. W. Tucker (Chairman), Einar Hille, J. R. Kline, and P. A. Smith as a committee to study problems of publication.

The following items were reported for the information of the Council: selection of G. T. Whyburn as Managing Editor of Transactions and Memoirs for 1951; A. W. Tucker as Chairman of Mathematical Surveys Editorial Committee for 1951; G. B. Price as Chairman of Bulletin Editorial Committee for 1951; Nathan Jacobson as Chairman of Proceedings Editorial Committee for 1951; R. L. Wilder as Chairman of Colloquium Editorial Committee for 1951; William Feller as Chairman of Mathematical Reviews Editorial Committee for 1951; as new members of the Policy Committee for Mathematics: E. H. C. Hildebrandt and Saunders MacLane (The Committee now consists of: Einar Hille, J. R. Kline (Chairman), W. T. Martin, and Marston Morse for the American Mathematical Society; H. M. Gehman, Saunders MacLane, and C. V. Newsom for the Mathematical Association of America; A. E. Meder for the Association of Symbolic Logic; Henry Scheffé for the Institute of Mathematical Statistics; and E. H. C. Hildebrandt for the National Council of Teachers of Mathematics); acceptance by Alfred Tarski of invitation to deliver the Colloquium Lectures at the Summer meeting of 1952; acceptance by Kurt Gödel of invitation to deliver the 25th Josiah Willard Gibbs Lecture at the Annual meeting, 1951; Committees to Select Hour 
Speakers have invited Ivan Niven, June meeting, Washington State College, and G. W. Whitehead, Summer meeting, Minneapolis to deliver addresses.

The following resolution on the death of Professor G. A. Miller was read and adopted by the Council:

The Council of the American Mathematical Society notes with regret the passing of George Abram Miller. Fifty-nine years ago last October Professor Miller was elected to membership in the New York Mathematical Society; of the present members of the American Mathematical Society only two preceded him. His membership was interrupted; he became a member again in 1898 and a life member in $1900 . \mathrm{He}$ was one of the founders of the San Francisco Section in 1902 and was its Secretary until 1906. He was a Vice President of the Society in 1908. Having returned to the Middle West, he was Chairman of the Chicago Section in 1908 and 1909.

During the years when he seems not to have been officially connected with the Society, he was carrying on the work to which the Society is dedicated. From 1893 to 1895 he was instructor at the University of Michigan. He lived in the home of F. N. Cole who was for so many years Secretary of the Society and who in 1891 was Secretary of the Mathematical Society of the University of Michigan. It was Cole's influence that started him on the study of finite groups which he followed throughout his life. From 1895 to 1897 he was at Leipzig and Paris. In Paris he became associated with C. Jordan who sponsored all his communications to the Comptes Rendus of the Société Mathématique de France.

Miller's first publications were two articles in volume 3 of the Bulletin of the New York Mathematical Society. By the end of the year of his return from Europe, he had published twenty-eight articles, fourteen of them in the Bulletin; by the end of 1915 he had contributed seventy-five articles to the Bulletin and the Transactions. He continued to contribute to the Bulletin until 1931 and to the Transactions until 1934. His last contribution to group theory, published in the Proceedings of the National Academy of Science, appeared in 1946 when he was eighty-three years old.

The Council salutes a man who was a pioneer in American mathematics, who brought prestige to the Society, and who for years enough was universally acknowledged as the leader in one branch of mathematics.

The Executive Director reported for the information of the Council on some of the problems arising in the central office. The late arrival of programs of meetings is being investigated.

The Executive Committee reported to the Council for its information that six bids had been received for the library and that the high bid of $\$ 66,000$, made by the University of Georgia, was the one best calculated to further the objectives of the Society, and that the Executive Committee had recommended to the Board of Trustees that this bid be accepted. (At their meeting on April 28, the Board of Trustees accepted this recommendation.) The Executive Committee also reported that after consideration of a number of possibilities it had recommended to the Board of Trustees that the Society lease a house from Brown University and move all its permanent offices there. (The Board of Trustees accepted this recommendation also.) 
Professor J. R. Kline reported for the Policy Committee and announced that the four governing boards had approved the addition of the National Council of Teachers of Mathematics to the Policy Committee; that a new Division of Mathematics has been created in the National Research Council with Marston Morse as chairman and M. H. Stone as vice chairman; that the United States will be represented in the International Mathematical Union by a U. S. National Committee for Mathematics with five members nominated by the Policy Committee and four by the Division of Mathematics.

The Council voted to grant a leave of absence to Professor J. W. Green as Associate Secretary for the academic year 1951-52 and appointed Professor W. T. Puckett to fill this position during Professor Green's absence.

The Council voted to approve the request of the Board of Trustees that the Associate Secretaries resume the arranging of programs of meetings.

The Council voted to authorize the President to appoint a committee to advise the Council in its reconsideration of its actions with regard to the University of California and to study the implications of these actions for the general policies of the Society.

The Council voted to invite Professor M. H. Stone to serve as the Society's Visiting Lecturer for 1951-1952.

The Council received the final report of the Birkhoff Memorial Finance Committee, which indicates that this project will approximately break even in the long run.

A meeting of the Southeast Section was set for November 23-24 at the Alabama Polytechnic Institute, Auburn, Alabama.

Abstracts of the papers presented are listed below, those with a " $t$ " after their numbers having been read by title. The joint papers numbered 268, 285, 291, 313, 316 were presented by Professor Brauer, Mr. Brock, Dr. Roberts, Dr. Hammer, and Dr. Chung, respectively. Dr. Arf was introduced by Professor Alexander Weinstein, Mr. Cherlin by Professor M. S. Robertson, and Mr. Ludford by Dr. Stefan Bergman.

\section{Algebra ANd Theory of Numbers}

267t. R. C. Bose and K. A. Bush: Upper bounds for orthogonal arrays.

Given an orthogonal array of strength $t$ and index $\lambda$, the question arises as to the maximum number of rows that can be constructed without destroying the orthogonality of the array. A number of such bounds are obtained which represent improvements over the bounds previously established by C. R. Rao. The improvements made are in general predicated upon certain questions of divisibility. Examples are 
given showing that these bounds may actually be attained in a number of cases. (Received March 12, 1951.)

\section{A. T. Brauer and T. L. Reynolds: On a theorem of Aubry-} Thue.

L. Aubry (Mathesis (4) vol. 3 (1913) pp. 33-35) and A. Thue (Det Kongelige Norske Videnskabers Selskab Skrifter (1915) no. 3 and Archiv for Mathematik og Naturvidenskab vol. 34 (1917) no. 15) proved independently that the Diophantine equation $a x-b y \equiv 0(\bmod m)$ has solutions for which $0<x \leqq m^{1 / 2},|y| \leqq m^{1 / 2}$. Vinogradov (Trans. Amer. Math. Soc. vol. 29 (1927) pp. 209-217) showed the existence of a solution for which $0<x \leqq k, 0 \leqq|y| \leqq m / k$ for any positive $k$. In this paper it is proved that a system of $r$ linear homogeneous congruences in $s$ unknowns $x_{1}, x_{2}, \cdots$, $x_{s}$ always has a nontrivial solution $\left|x_{\sigma}\right| \leqq m^{r / s}(\sigma=1,2, \cdots, s)$. Similarly Vinogradov's theorem is generalized. The theorem of Aubry-Thue was used in particular for a simple proof of the representation of the primes of form $4 n+1$ as sum of two squares and for the proof that the least $k$ th power nonresidue $\bmod p$ with $p \equiv 1(\bmod k)$ is less than $p^{1 / 2}$ for odd $k$. Correspondingly the generalization gives a simple proof for the four-square theorem. Moreover, it follows that each of the $k-1$ classes of $k$ th power non-residues contains elements less than $p^{(k-1) / k}$. (Received March 14, 1951.)

\section{9t. K. A. Bush: Orthogonal arrays of strength 2.}

An orthogonal array of strength 2 is a rectangular array with the properties: i. Each row consists of the integers $0,1,2, \cdots, s-1$ each repeated $s$ times. ii. Each of the $s^{2}$ possible ordered pairs that can be formed from the integers $0,1,2, \cdots, s-1$ occurs exactly $\lambda$ times when all pairs of the form $\left(a_{i k}, a_{j k}\right)$ are constructed, where $a_{i k}$ is the element in the $i$ th row and $k$ th column of the array and where $k$ ranges over the $s^{2}$ columns of the array. This property holds for all choices of $i$ and $j, i \neq j$. The construction of such arrays is effected by use of Galois fields where $s=p^{u}$ and $\lambda=p^{v}$ and $p$ is a prime. This generalizes certain earlier results such as the case when $v=0$ (identical with the problem of constructing a complete set of orthogonal Latin squares) and a more recent result due to R. A. Fisher. (Received March 12,1951.)

\section{K. A. Bush: Orthogonal arrays of index unity and strength $t$.}

Generalizing the ideas of the preceding abstract, we may consider ordered $t$-plets by selecting $t$ rows from the orthogonal array. There are $s^{t}$ such $t$-plets. If independently of the $t$ rows selected from the array, every $t$-plet occurs exactly once, we say we have an orthogonal array of index unity, that is, $\lambda=1$, and strength $t$. In case $t$ exceeds $s$, it is possible to construct $t+1$ such rows. In the nontrivial case where $s$ exceeds $t$, it is possible to construct $s+1$ orthogonal rows using polynomials in a finite geometry if $s$ is a prime or a power of a prime. For the particular case $t=3, s=2^{m}$ it is possible to improve this result, and here $s+2$ orthogonal rows can be constructed. This anomaly is due to the fact that a certain matrix of Vandermonde type is singular for this case. (Received March 12, 1951.)

\section{1t. Leonard Carlitz: $A$ conjecture of Dickson.}

Let $p$ be an odd prime. Dickson (Trans. Amer. Math. Soc. vol. 10 (1909) pp. 109122 ) considered binary forms $Q(x, y)$ with coefficients in $G F\left(p^{n}\right)$ such that $Q(a, b)$ is a nonzero square in $G F\left(p^{n}\right)$ for all $(a, b)$ except $(0,0)$ in the field. He conjectured that every such form of degree $2 r, r>1$, is the square of a form provided $p^{n}$ exceeds a 
certain bound $N_{r}$. In a previous note (Duke Math. J. vol. 14 (1947) pp. 1139-1140) the writer showed that this conjecture is a consequence of A. Weil's theorem on the zeros of the Artin zeta function. In the present note it is shown that the result can be obtained without the use of Weil's theorem. It suffices to make use of a theorem of Davenport (Acta Math. vol. 71 (1939) pp. 99-121, Theorem 4); however this method leads to a larger bound $N_{r}$. (Received March $\left.7,1951.\right)$

\section{A. B. Clarke: Directed sums of cardinal algebras.}

A definition of a directed sum of cardinal algebras (C.A.'s) is given, analogous to the definition for partially ordered sets. Necessary and sufficient conditions are given for such a directed sum to be a C.A., and it is shown that the only essentially nontrivial case is when the index algebra is idemmultiple and linearly ordered. Every C.A. has a unique representation as such a linear directed sum of C.A.'s which are indecomposable in this way. A C.A. is called primary if $(\infty a<\infty b \rightarrow a<b)$. By application of the preceding theorem it is shown that every primary C.A. can be represented as a directed sum of C.A.'s which are idemmultiple or simple. An operation on C.A.'s is defined which is a generalization of finite direct product, and the class of all C.A.'s that can be obtained from primary C.A.'s by this operation is characterized. To round out the discussion it is shown that a necessary and sufficient condition for a simple C.A. to be a subalgebra of the non-negative reals is $(a \neq 0, b$ finite $\rightarrow b \leqq n a$ for some $n<\infty)$. (Received March 13, 1951.)

\section{C. W. Curtis: Ideal theory in noncommutative rings. Prelimi-} nary report.

Generalizations of primal and primary ideals are given for two-sided ideals in a noncommutative ring $R$ with an identity, and applied, in case $R$ satisfies the maximum condition for ideals, to discuss the additive ideal theory in $R$, completing and extending in some respects the work of Fuchs (Proceedings of the American Mathematical Society vol. 1 (1950) pp. 1-6) and Krull (Math. Zeit. vol. 28 (1928) pp. 481-503). The existence of representations of ideals as intersections of primal ideals is proved for arbitrary rings, and the uniqueness is investigated when $R$ satisfies the maximum condition. The existence of primary representations is proved for certain classes of rings. For example, one class consists of all polynomial (or power series) rings in $n$ commuting indeterminates with coefficients in a central simple algebra; another class consists of all rings with maximum condition having the property that every ideal is two-sided. The theory of primary ideals is used to study the powers of an ideal. It follows from this discussion and a result of Jacobson (Amer. J. Math. vol. 67 (1945) pp. 300-320) that if every ideal in $R$ is an intersection of primary ideals, and if every ideal in $R$ has a finite basis as a right ideal, then the intersection of the powers of the Jacobson radical of $R$ is zero. (Received March 9, 1951.)

274. Jesse Douglas: On the existence of a basis for every finite abelian group.

Every element $x$ of a finite abelian group $G=a, b, c, \cdots, z$ (of order $g$ ) can be expressed as a product of powers of a properly chosen subset of elements, called a basis: for example, $x=b^{\beta} a^{\delta} l^{\lambda} r^{\rho}$, the exponents being uniquely determined modulo the corresponding periods $\beta_{0}, \delta_{0}, \lambda_{0}, \rho_{0}\left(g=\beta_{0} \delta_{0} \lambda_{0} \rho_{0}\right)$. The proofs of this fundamental theorem of abelian groups given in the literature are fairly complicated, involving: H. Weber (1899), mathematical induction; R. Fricke (1924), cosets and quotient 
groups; L. Pontrjagin (1946), matrices. The present paper offers a proof superior in simplicity and perspicuity. Supposing first $G$ of prime power order, $g=p^{h}$, let us arrange the elements of $G$ in a nonascending order of their periods: $p^{m}, p^{m-1}, p^{m-2}$, $\cdots, p, 1(m \leqq h)$. Then, running through the elements in this order, remove successively each element equal to a power product of those yet remaining, distinct from itself. What is left will be a basis of $G$. The extension to the general case-where $g=p^{h} q^{k} \cdots r^{l}, p, q, \cdots, r$ being different primes-is easy, resting on the standard fact that the abelian group $G$ is then the direct product of abelian groups of the respective prime power orders: $p^{h}, q^{k}, \cdots, r^{l}$. The complete paper will appear in an early issue of Proc. Nat Acad. Sci. U.S.A. (Received April 28, 1951.)

\section{5t. Gerald Freilich: Ideals in rings of transformations.}

A complete characterization of two-sided ideals in the ring $\mathcal{L}$ of all linear transformations of a vector space $V$ into itself is given. If $F$ is a two-sided ideal in $\mathcal{L}$, then it is proved that $F=\{\alpha \in \mathcal{L} \mid \operatorname{dim}[\alpha(V)]<\boldsymbol{N}\}$ for some cardinal $\mathbf{N}$. There is further established a correspondence between one-sided ideals in $\mathcal{L}$ and sets of subspaces of $V$. By considering retractions in dense subrings of $\mathcal{L}$, a theory for left ideals similar to Jacobson's theory of right ideals is established (see N. Jacobson, Structure theory of simple rings without finiteness assumptions, Trans. Amer. Math. Soc. vol. 57 (1945) pp. 228-245). Finally, an example is given of a dense ring of linear transformations containing no nontrivial finite-valued transformation. (Received February 6, 1951.)

\section{R. E. Johnson: The extended centralizer of a ring over a module.}

For the ring $R$ and the right $R$-module $M$, let $\mathfrak{M}$ denote the set of all submodules $N$ of $M$ having the property that $N \cap N^{\prime} \neq 0$ for all nonzero submodules $N^{\prime}$ of $M$. An $R$-homomorphism of any submodule $N$ of $M$ into $M$ is called a semi-endomorphism of $M$. In the set $\Re$ of all semi-endomorphisms of $M$ operating on elements of $\mathfrak{M}$, operations of addition and multiplication are introduced. Relative to these, $\Omega$ has ring-like properties. The extended centralizer of $R$ over $M$ is $\Re / \mathfrak{S}$, where $\mathfrak{S}$ is the set of all semi-endomorphisms of $M$ having annihilator in $\mathfrak{M}$. The extended centralizer of $R$ over $M$ is shown to be a regular ring. By letting $M=R$, necessary and sufficient conditions are obtained for a ring $R$ to have a regular quotient ring. (Received March 12, 1951.)

\section{7t. Jakob Levitski: Remarks on the determinant $\left[x_{1}, x_{2}, \cdots, x_{r}\right]$.}

Consider the "determinant" $\left[x_{1}, x_{2}, \cdots, x_{r}\right]=\sum_{(i)} x_{i_{1}} x_{i_{2}} \cdots x_{i_{r}}$, where $(i)$ is an arbitrary permutation of $r$ letters, and the sign is positive for even permutations and negative for odd permutations. Let $A$ denote the total metric algebra $F_{n}$ of the $n$ by $n$ matrices over a field $F$. Denote further by $A_{0}$ the linear space over $F$ of all matrices having a zero trace, and by $M_{r}$ the linear space over $F$ spanned by the totality of the determinants $\left[a_{1}, a_{2}, \cdots, a_{r}\right]$ formed for all possible sequences of $r$ matrices $a_{1}, a_{2}, \cdots, a_{r}$. It has been recently shown (A. S. Amitsur and J. Levitzki, Proceedings of the American Mathematical Society vol. 1 (1950)) that $M_{r}=0$ if and only if $r \geqq 2 n$. In the present note we determine $M_{r}$ for $r<2 n$. Evidently $M_{1}=A$ and it is known that $M_{2}=A_{0}$. We generalize these facts by showing that if $2 m<n$ then $M_{2 m}=A_{0}$, while for $2 m+1<2 n$ and if the characteristic of $F$ is zero we have $M_{2 m+1}=A$. This equality holds also in case $F$ has a positive characteristic $p$ which is not a factor of $2 m+1$. In case $p$ is a factor of $2 m+1$ we have the equality $M_{2 m+1}=A_{0}$. These results remain valid for general central simple algebras of finite dimension. (Received February 16, 1951.) 


\section{8t. R. C. Lyndon: Identities in groups, semigroups, and algebras.}

An identity in an abstract algebra is an equation in indeterminates that holds true under every substitution of elements from the algebra. Results: (1) All identities in a nilpotent group of class $n$ are derivable from a finite set of identities involving no more than $n$ indeterminates; (2) All identities in a two-element algebra are derivable from some finite set of identities; (3) There exists an infinite semigroup (without cancellation) whose identities are not derivable from any finite set. Problem: Does every finite abstract algebra have a finite set of identities from which all others are derivable? (Received March 15, 1951.)

\section{Maxwell Rosenlicht: Equivalence relations on algebraic curves.}

The structure of an arbitrary subring of an algebraic function field of one variable over an arbtirary ground field is analyzed in detail. If, in particular, one deals with a subring that is semilocal in the sense of Chevalley, then the analysis amounts to the study of the singularities of a suitable projective model of the field. In this case an equivalence relation among divisors of the field can be defined by setting equivalent to the unit divisor those divisors that are divisors of units in the semilocal ring; this generalizes the classical linear equivalence of divisors. A generalized Riemann-Roch theorem is then proved, in which corresponding to the ordinary differentials of the first kind appear differentials having certain types of singularities at certain places of the field. In the case where the ground field is the complex numbers, one obtains generalizations of Abel's theorem and the Jacobi inversion theorem; in the abstract case one has new Jacobian varieties. The theory affords a very simple proof of the theorem of Gorenstein on the conductor of the local ring of a point on a plane curve. (Received March 6, 1951.)

\section{R. D. Schafer: Representations of alternative algebras.}

Eilenberg's definition of representation (Annales de la Société Polonaise de Mathématique vol. 21 (1948) pp. 125-134) is applied to alternative algebras. A generalization to alternative algebras of characteristic 0 of the Whitehead first lemma for associative algebras is obtained as a corollary of Jacobson's analogous result for Jordan algebras. However, a stronger form of this lemma is required in the proof of the generalization to alternative algebras of Malcev's theorem on the strict conjugacy of semisimple components in Wedderburn decompositions. A related theorem states that an alternative algebra (of characteristic 0 ) is semisimple if and only if its derivation algebra is semisimple or $\{0\}$. (Received February 26,1951.)

\section{1t. J. A. Silva: A theorem on cyclic determinants.}

In this paper the following generalization of a well known result on cyclic determinants is given. Let $A$ be a cyclic matrix of order $n^{2}$ given by $A=\left\|a_{j-i+1}\right\|$, $i, j=1,2, \cdots, n, a_{r}=a_{s}$ for $r \equiv s(\bmod n)$. Let $p$ be a rational prime such that $n=p^{t} m$, $p \nmid m$. Then $d(A) \equiv \prod_{i=1}^{m}\left(\sum_{j=1}^{n} \alpha_{i}^{j-1} a_{j}\right)^{p^{t}}(\bmod p)$, where the $\alpha_{i}$ run through the $m$ distinct $n$th roots of unity $(\bmod p)$. (Received March 12,1951.)

\section{ANALysis}

\section{Cahit Arf: On Rayleigh-Ritz-Weinstein's method.}

The author considers a self-adjoint continuous operator $Q$ which is such that there exists a number $\zeta$ and a continuous self-adjoint operator $R_{\zeta}$ for which $R_{\zeta}^{\prime}(Q-I \zeta)$ 
$=(Q-I \zeta) R_{\xi}^{\prime}=\bar{P}_{\zeta}$ is a projector which satisfies the condition $(Q-I \zeta)\left(I-\bar{P}_{\zeta}\right)=0$. The main known results about Weinstein's determinant are extended to those operators which satisfy the conditions stated above. The subspaces considered in this extension do not necessarily differ from the whole space by a finite space. (Received April 19, 1951.)

\section{3t. Stefan Bergman: On the solution of the initial value problem for equations of mixed type.}

For the differential equation $\psi_{\mathrm{HH}}+l(\mathrm{H}) \psi_{\theta \theta}=0, l(\mathrm{H})>0$ for $\mathrm{H}<0, l(\mathrm{H})<0$ for $\mathrm{H}>0$, the solution of the initial value problem in the small is considered for data given on the transition line. This is done by means of the set of particular solutions introduced in a paper by the author (Bergman, Proc. Nat. Acad. Sci. U.S.A. vol. 29 (1943) p. 276), $\psi_{n, 1}(\mathrm{H}, \theta)=1 ! C_{n, 1} \theta^{n-1} \int_{0}^{\mathrm{H}^{\mathrm{H}}} d \mathrm{H}_{1}-3 ! C_{n, 3} \theta^{n-3} \int_{0}^{\mathrm{H}} d \mathrm{H}_{3} \int_{0}^{\mathrm{H}_{3}} l\left(\mathrm{H}_{2}\right) d \mathrm{H}_{2} \int_{0}^{\mathrm{H}_{2}} d \mathrm{H}_{1}$, $\psi_{n, 2}(\mathrm{H}, \theta)=\theta^{n}-2 ! C_{n, 2} \theta^{n-2} \int_{0}^{\mathrm{H}} d \mathrm{H}_{2} \int_{0}^{\mathrm{H}_{2}} l\left(\mathrm{H}_{1}\right) d \mathrm{H}_{1}+\cdots(n=1,2, \cdots)$. The following theorem holds. Let the functions $\chi_{k}(\theta)(k=1,2)$ possess expansions of the form $\chi_{k}(\theta)=\sum_{\nu_{n=0}}^{\infty} A^{(k)} \theta^{\nu},|\theta| \leqq \theta_{1} . \psi(\mathrm{H}, \theta)=\sum_{n=1}^{\infty} n^{-1} A_{n+1}^{(2)} \psi_{n, 1}(\mathrm{H}, \theta)+\sum_{n=0}^{\infty} A_{n}^{(1)} \psi_{n, 2}(\mathrm{H}, \theta)$ then converges in $|\theta|+\sigma|\mathbf{H}|<\theta_{1}$, for some $\sigma>0$, and represents a solution of the above differential equation for which $\psi(0, \theta)=\chi_{1}(\theta), \psi_{\mathrm{H}}(0, \theta)=\chi_{2}(\theta)$. (Received March 19, 1951.)

284t. R. H. Breusch: On the distribution of the roots of a polynomial with integral coefficients.

D. H. Lehmer (Ann. of Math. vol. 34 (1933) p. 476) has proposed the following problem: to find irreducible, noncyclotomic polynomials $f(z) \equiv z^{r}+a_{1} z^{r-1}+\cdots \pm 1$ with integral coefficients, such that $\Omega(f)$, the product of the absolute values of those roots of $f(z)$ which lie outside the unit circle, shall be as small as possible. In the present note it is shown that for nonreciprocal polynomials $\Omega(f)>1.179$. The proof makes use of the fact that $R(f, g)$, the resultant of $f(z)$ and $g(z) \equiv z^{r} \cdot f(1 / z)$, is absolutely not less than 1 . (Received March 9, 1951.)

285. Paul Brock and F. J. Murray: The use of exponential sums in step by step integration.

R. E. Greenwood has suggested the use of exponential sums instead of polynomials in the step by step integration of a system of differential equations (Ann. Math. Statist. vol. 20 (1949) p. 608-611). In the present paper it is shown that if an "open" method of integration is precise for a step $h$ and exponents $v_{1}, \cdots, v_{n}$, then the error in integrating the function $e^{\lambda t}$ for a $t$ step of length $h$ is in the form $h \epsilon(\lambda h) e^{\lambda t}$ where $\epsilon(\lambda h)$ is given by the following. Let $y_{j}=e^{-v j h}-1, y=e^{-\lambda h}-1$, let $S_{k}$ denote the sum of the monomials of degree $k$ in $y, y_{1}, \cdots, y_{n}$, and let $A_{l}$ be defined by the equation $y(1+y)^{-1}(\log (1+y))^{-1}=\sum_{l} A_{l} y^{l}$. Then $\epsilon(\lambda h)=(-1)^{n+1} \prod_{i=1}^{n}\left(y-y_{j}\right)\left(\sum_{l=0}^{\infty} A_{n+l} S_{l}\right)$. For $\lambda h$ and $v_{j} h$ small this behaves like $-h^{n} \pi\left(\lambda-v_{j}\right)$ and thus the step by step error goes to zero like $h^{n+1}$. In order to take care of the case in which the integrand is equal to a linear combination of exponential functions and a small residual function $\sigma(t)$, the error for an arbitrary integrand, $\sigma(t)$, is also studied. (Received March 12, 1951.)

286t. Felix Browder: On isolated singularities of second-order linear elliptic partial differential equations.

Let $L(u)=A u_{x x}+2 B u_{x y}+C u_{y y}+D u_{x}+E u_{y}+F u=G$ be the general second-order 
linear elliptic partial differential equation whose coefficients are defined in a neighborhood $N$ of a point $P$ in $E^{2}$ and such that $A, B, C$ are three-times continuously differentiable in $N, D$ and $E$ twice continuously differentiable, $F$ continuously differentiable and $G$ continuous in $N .\left(A C-B^{2}>0\right.$.) Suppose $u$ is a regular solution of $L(u)=0$ in $N-\{P\}$ such that $\int_{N}\left(u_{x}^{2}+u_{v}^{2}\right) d A<\infty$. Then the singularity of $u$ at $P$ is removable. More generally let $L(u)=\Delta u+\sum_{j=1}^{n} b^{i} u_{x} j+c u=d$ be a second-order linear partial differential equation of elliptic type in a neighborhood $N$ of a point $P$ in $E^{n}$, the $n$-dimensional Euclidean space, where $b^{i} \in C^{2}(N), c \in C^{1}(N), d \in C^{0}(N)$ and $\Delta$ is the Laplace operator $\sum_{i=1}^{n} \partial^{2} / \partial x_{i}^{2}$. Suppose $u$ is a regular solution of $L(u)=0$ in $N-\{P\}$ such that $\int_{N}\left(\sum_{j_{n=1}}^{n=1} u_{x}^{2}\right) d A<\infty$. Then the singularity of $u$ at $P$ is removable. Weaker conditions in terms of the rate of growth of the Dirichlet integral near $P$ are formulated which insure the removability of the singularity when the latter integral is divergent. (Received March 15, 1951.)

\section{7t. K. A. Bush: Continuous functions possessing no derivative.}

A class of continuous functions is constructed with the interesting property that $f(x)-k=0$ possesses a nondenumerable number of solutions where $f(x)$ is a member of the class and $k$ is any constant lying between the infimum and supremum of $f(x)$. These solutions form a set of measure zero, and these functions fail to have a derivative anywhere. (Received March 12, 1951.)

288t. G. Y. Cherlin: Bounds for the coefficients of some classes of odd multivalent functions.

Let $f(z)=\sum_{0}^{\infty} a_{2 n+1} z^{2 n+1}$, an odd function, be regular for $|z|<1$ with the property that for a given positive integer $p$ (which must be odd) and a given complex number $\alpha$ the imaginary part of $\alpha f(z)$ has exactly $2 p$ changes of sign on $|z|=r$ for a range $1-\epsilon<r<1$. Then a bound for all the coefficients is given in terms of the first $(p+1) / 2$ coefficients. The proof is by induction following a modification of the method of A. W. Goodman and M. S. Robertson [Trans. Amer. Math. Soc. vol. 70 (1951) pp. 127-136]. Application is made to a coefficient problem of multivalent odd functions of order $p$. (Received March 12, 1951.)

289t. V. F. Cowling and George Piranian: On the summability of ordinary Dirichlet series by Taylor methods.

Let $V_{\alpha}$ be the series-to-series transformation $V_{\alpha} \sum x_{n}=\sum y_{n}$ represented by the upper-triangular matrix $\left(v_{n k}\right)$ with $v_{n k}=(1-\alpha)^{n} C_{k, n} \alpha^{k-n}$ for $k \geqq n$. The authors establish the following result: if the series $\sum a_{n}(n+1)^{-s}$ is summable by $V_{\boldsymbol{\alpha}}$ at $s_{0}$, and the parameter $\alpha$ is suitably restricted, then the series is absolutely summable by $V_{\alpha}$ in the half-plane $\Re s>1+\Re s_{0}$; if the summability at $s_{0}$ is absolute, the series is absolutely summable in the half-plane $\Re s>\Re s_{0}$. (Received April 9, 1951.)

290. J. B. Diaz: A remark on the method of Trefftz and on its generalizations.

It is shown that a lower bound for the Dirichlet integral $D(u)=\int_{R}\left(u_{x}^{2}+u_{y}^{2}\right) d x d y$ of the solution $u$ of Dirichlet's problem: $\Delta u=0$, on $R, u=$ a given function, on $C$ (where $R$ is a plane domain and $C$ its boundary), is given by $(*): D(\alpha w) \leqq D(u)$, where $w$ is an arbitrarily chosen nonconstant function (possibly multiple-valued, but such that $w_{x}$ and $w_{y}$ are single-valued) and $\alpha=\left(\int c u(\partial w / \partial s d s)(D(w))^{-1}\right.$. The inequal- 
ity $(*)$ generalizes a classical result of E. Trefftz (Proceedings of the Second International Congress of Applied Mechanics, Zürich, 1927, p. 131), which states that $D(\beta h) \leqq D(u)$, where $h$ is required to be a nonconstant harmonic function and $\beta=\left(\int_{C} u(\partial h / \partial n) d s\right)(D(h))^{-1}$. The inequality $(*)$ corresponds to the lower bound for the Dirichlet integral in Neumann's problem, which was recently given, also in terms of an arbitrary nonconstant function (J. B. Diaz and A. Weinstein, Journal of Mathematics and Physics vol. 26 (1947) p. 133, and Amer. J. Math. vol. 70 (1948) p. 107; see also the article by A. Weinstein, Proceedings of Symposia in Applied Mathematics vol. 3, 1950, p. 141). Similar results hold for any number of dimensions. (Received January 18, 1951.)

291. J. B. Diaz and R. C. Roberts: On the numerical solution of the Dirichlet problem.

The convergence of several computational methods for the solution of the difference equation form of the classical Dirichlet problem in a bounded domain is considered. In particular, it is shown that the precise analogues of Poincarés "methode de balayage" and of its extension by O. D. Kellogg (in his book, Potential theory, Berlin, 1929) yield sequences of functions which converge to the solution of the difference boundary value problem. In the usual proofs of the convergence of successive approximations to the solution of the difference boundary value problem (see, for example, P. Frank and R. v. Mises, Differential und Integralgleichungen der Mechanik und Physik, New York, 1943, p. 734, and I. G. Petrovskii, Lectures on partial differential equations, Moscow, 1950, p. 282) a definite ordering of the points of the domain is essential. By the present method, such a preliminary ordering of the points is seen to be entirely unnecessary. The relationship between the "methode de balayage" and the relaxation method of numerical approximation is also clarified. (Received March 14, 1951.)

\section{J. A. Dieudonné: Sequences of convergent Radon measures.}

Let $\left(f_{n}\right)$ be a sequence of Lebesgue integrable functions on the closed interval $(0,1)$. It is well known that if $\int_{A} f_{n}(x) d x$ tends to a limit for every Borel set $A$ in the interval, then there is an integrable function $f$ such that, for every bounded and measurable function $g, \int f_{n}(x) g(x) d x$ tends to $\int f(x) g(x) d x$. It is proved that the same conclusion is true if it is assumed only that $\int_{A} f_{n}(x) d x$ tends to a limit for every closed set $A$ in the interval. The result can be extended to any Radon measure on a compact space. (Received March 12, 1951.)

\section{3t. Herbert Federer: Essential continua of surfaces. I.}

Suppose $f$ is a map of the closed $k$-cell $X$ into $n$-space with finite Lebesgue area $L(f)$. The class $Z$ of all maximal continua of constancy of $f$ is metrized so that the distance between $A$ and $B$ in $Z$ is the infimum of the diameters of the $f$ images of those connected subsets of $X$ which meet both $A$ and $B$. To this metric corresponds the $k$-dimensional Hausdorff measure $H$ over $Z$. Let $f=l m$ be the monotone-light factorization of $f$ with middle space $Z$. For every positive number $r$, each continuum $A$ in $Z$ is a subset of a unique component $U(A, r)$ of the $f$ counterimage of the closed sphere in $n$-space with center $l(A)$ and radius $r . L^{*}(A)$ and $L_{*}(A)$ are the upper and lower limits, as $r$ approaches zero, of $L(f \mid U(A, r)) /\left(w r^{k}\right)$, where $w$ is the volume of a unit sphere in $k$-space. Let $H^{\prime}$ be the ordinary $k$-dimensional Hausdorff measure over $n$-space. It is shown that $L(f) \geqq \int L^{*}(A) d H A \geqq \int \sum_{l(A)=y} L^{*}(A) d H^{\prime} y$. (Received February 5,1951 .) 


\section{4t. Herbert Federer: Essential continua of surfaces. II.}

Let $s(g)$ be the $k$-dimensional Lebesgue measure of the set of all stable values of any mapping $g$ into $k$-space. An orthogonal projection $p$ of $n$-space onto $k$-space is essential at a point $A$ of $Z$-and $A$ is an essential continuum of $f$-if and only if $\lim _{r \rightarrow 0} s(p f \mid U(A, r)) /\left(w r^{k}\right)=1$. This is shown to imply $\lim _{r \rightarrow 0} s(p f \mid T \cap U(A, r)) /\left(w r^{k}\right)$ $=1$ whenever $t<1$ and $T=\{x|t \cdot| f(x)-l(A)|\leqq| p f(x)-p l(A) \mid\}$. The number of projections which are essential at $A$ does not exceed $L_{*}(A)$, and the set $E$ of all essential continua of $f$ has the finite measure $H(E) \leqq \int L_{*}(A) d H A$. If $L(f)=\int L^{*}(A) d H A$ and $H\left(\left\{A \mid L^{*}(A)>0\right\}\right)$ is finite, then $H$ almost all points of $E$ possess unique essential projections, $l(E)$ is an $\left(H^{\prime}, k\right)$ rectifiable subset of $n$-space (Trans. Amer. Math. Soc. vol. 62 (1947) pp. 114-192), whose $H^{\prime}$ approximate tangent planes correspond to the essential projections, and $H(E)=\int N(y) d H^{\prime} y$, where $N(y)$ is the number (possibly $\infty$ ) of points $A$ in $E$ for which $l(A)=y$. If $k=2$, all this holds with $L(f)=H(E)$, the projection properties of $f$ are those of the set $l(E)$ which $H^{\prime}$ almost lies on countably many continuously differentiable surfaces, and surface integrals over $f$ are computable by applying the classical formalism to $l(E)$. All objects considered in this paper are proved to be Fréchet covariants of $f$. (Received February 5, 1951.)

295. R. S. Finn: On sequences of solutions of quasi-linear elliptic equations. Preliminary report.

Quasi-linear elliptic partial differential equations of the form $(*)\left(\rho \phi_{x}\right)_{x}+\left(\rho \phi_{y}\right)_{y}=0$ are considered. The function $\rho=f\left(x, y, \phi_{x}^{2}+\phi_{y}^{2}\right)$ is to satisfy the conditions: (a) $A_{1} \omega^{-\theta}$ $<f\left(x, y, \omega^{2}\right)<A_{2}$, (b) $f\left(x, y, \omega^{2}\right)+2 \omega^{2} \partial f\left(x, y, \omega^{2}\right) / \partial \omega^{2}>\omega^{-m}$, for $0<\theta<1, A_{1}>0$, $A_{2}>0, m>0$, and $\omega$ sufficiently large. It is assumed that $f\left(x, y, \omega^{2}\right)$ satisfies certain regularity conditions. The class of equations thus defined includes the minimal surface equation. Let $\left\{\phi_{n}(x, y)\right\}$ be a sequence of solutions of $(*)$ regular in a domain $D$ and continuous in $D+D^{\prime}$, which converge uniformly on $D^{\prime}$. Then $(1)\left\{\phi_{n}(x, y)\right\}$ converges uniformly in $D+D^{\prime}$ to a continuous function $\phi_{0}(x, y)$, (2) there exists in $D$ an open set $U$ equal in measure to $D$ and a subsequence $\left\{\phi_{n_{k}}\right\}$ whose derivatives up to the second order converge uniformly to those of $\phi_{0}(x, y)$ on every closed subset of $U$. It follows [see Morrey (Trans. Amer. Math. Soc. vol. 43 (1938) pp. 126-166), also Schauder (Math. Zeit. vol. 37 (1933) pp. 623-634)] that if $D$ is convex and if $\rho=f\left(\phi_{x}^{2}+\phi_{y}^{2}\right)$, then there exists a continuous function $\phi(x, y)$ which is a solution of $(*)$ throughout $D$ except on a set $E$ whose closure $\bar{E}$ is of measure zero, and which assumes on $D^{\prime}$ arbitrarily prescribed continuous values. It is shown that the set $\bar{E}$ is composed of continua connected to $D^{\prime}$. (Received March 15, 1951.)

296. B. M. Ingersoll: On a problem of Cauchy type for linear hyperbolic equations. Preliminary report.

The equation $L(u)=u_{x y}+a u_{x}+b u_{y}+c u=f$ is considered. Let $C$ be a smooth arc which is nowhere tangent to a characteristic and $R$ the Riemann rectangle determined by $C$. Two mixed derivatives of arbitrary orders are assigned on $C$, and values of a solution at $N$ points of $R$, where the integer $N$ depends on the orders of the derivatives. The coefficients of $L(u)$ are assumed to have certain regularity properties which, again, depend on the orders of the derivatives. It is shown that the above problem is correctly set, in general. The choice of the $N$ points is not entirely arbitrary. This gives rise to certain geometrical configurations, among which are curves which correspond to conjugate points in $N$ point problems for ordinary linear differential equations of $N$ th order. Exceptional cases, which arise from relations among the orders of the assigned derivatives, reveal certain phenomena. Among these is the 
existence of new characteristics, which depend only on $a$ and $b$, and along which the prescribing of two derivatives does not determine a solution-or, at least, not uniquely. Moreover, in certain cases, apparently $C$ must satisfy higher order differentiability conditions. (Received February 23, 1951.)

\section{7t. P. D. Lax: Completely continuous transformations. Prelimi- nary report.}

Let $B$ be a Banach space within which a continuous scalar product $(x, y)$ is defined, that is, $(x, y)$ is a bilinear skew-symmetric positive definite functional, with $(x, x)^{1 / 2} \leqq$ const $\|x\|$. Let $T$ be a transformation in $B$ which is symmetric with respect to this scalar product. Then: if $T$ is bounded in $B$, it is bounded with respect to the metric $(x, x)^{1 / 2}$, and if $T$ is completely continuous in $B$, it is completely continuous in the metric $(x, x)^{1 / 2}$ and the eigenelements of $T$ in $B$ with nonzero eigenvalue span the image of $B$ under $T$ in the sense of the metric $(x, x)^{1 / 2}$. This result is applied to formal self-adjoint second order elliptic operators $M$ in $m$ dimensions by noting that $v=T u$, defined as the solution of $M v=u, v=0$ on boundary, is completely continuous in the sense of the maximum norm, and symmetric with respect to an appropriate scalar product. (Received January 31, 1951.)

298. Benjamin Lepson: On exceptional sets of regular hyperdirichlet series. Preliminary report.

Certain necessary measure theoretic properties of these sets have been given previously by the author. It is shown here, using a known unpublished analog for capacity of Egoroff's theorem, that the sets are of logarithmic capacity zero. The sufficiency question is attacked, and a partial result is contained in the following theorem. Let $S$ be a closed bounded set of transfinite diameter zero. Then there exists a regular hyperdirichlet series which is $M$-convergent on $S$ but whose half-plane of termwise boundedness does not intersect $S$. (Received March 14, 1951.)

299t. Albert Novikoff: Integral representations for Legendre polynomials.

Integral representations, analogous to the formulae of Laplace and DirichletMehler for Legendre polynomials, are obtained for the polynomials of PollaczekSzegö, defined by the generating function $\left(1-z e^{i \theta}\right)^{\lambda_{1}+i \phi(\theta)}\left(1-z e^{-i \theta}\right)^{\lambda_{2}-i \phi(\theta)}$ $=\sum_{0}^{\infty} z^{n} R_{n}^{\left(\lambda_{1}, \lambda_{2}\right)}(\cos \theta ; a, b), \phi(\theta)=(a \cos \theta+b) / 2 \sin \theta, a \geqq \pm b$. Using the saddle-point method, the Laplace representation is made to yield an estimate of $R_{n}\left(\cos t / n^{1 / 2}\right)$, for large $n$. This estimate reveals a fundamental disparity between the PollaczekSzegö polynomials and the customary generalizations of the Legrendre polynomials with algebraic weight functions. It is shown that the zeros of $R_{n}\left(\cos t / n^{1 / 2}\right)$ all cluster at $t=(a+b)^{1 / 2}$ for $n$ large, and hence the associated limiting function $\lim _{n \rightarrow \infty} n^{1 / 4}$ $\cdot e^{((a+b) / 2)\left(1+n^{1 / 2}\right)} R_{n}\left(\cos t / n^{1 / 2} ; a, b\right)=S(t ; a, b)$, which would play the role of the Bessel functions for Jacobi polynomials, is seen to have an essential singularity at $(a+b)^{1 / 2}$. (Received March 14, 1951.)

\section{0t. R. S. Phillips: Spectral theory for semi-groups of linear oper- ators.}

A given semi-group of operators $T(t)$ on a Banach space $X$, strongly continuous for $t>0$, is embedded in a commutative Banach algebra $R$. According to the Gelfand theory, each $B \in R$ can be represented as a continuous complex-valued function $B(m)$ 
over the maximal ideals $M$ of $R$. A semi-group $T(t)$ is uniformly continuous at zero if and only if $T(t)(m)$ is of the form $\exp [a(m) t]$ on $M$. If the spectrum $\sigma[T(t)]$ for some interval $0 \leqq a<t<b$ contains no point of a connected open set containing 0 and $\infty$, then $T(t)$ has a bounded infinitesimal generator $A$. For a measure function $\alpha$, we define $\Theta(\alpha) x=\int_{0}^{\infty} T(t) x d \alpha$ and $f_{\alpha}(\lambda)=\int_{0}^{\infty} \exp (-\lambda t) d \alpha$. If $T(t)$ is uniformly continuous for $t \geqq c>0$, then $\sigma[\Theta(\alpha)]=f_{\alpha}[\sigma(A)] \cup_{\alpha}(0)$ and this correspondence holds as well for the detailed spectra. If $T(\zeta)$ is also analytic sufficiently far out in some sector, $\phi_{1}<\arg \zeta<\phi_{2}$, and if we define $\sigma(\phi)=\lim _{r \rightarrow \infty} r^{-1} \log \|T[r \exp (i \phi)]\|$, then $\sigma(\phi)$ is the function of support for the closed convex extension of the complex conjugate to $\sigma(A)$. The method is also applied to obtain a new and direct proof of the Stone theorem for locally compact groups of unitary transformations on a Hilbert space. (Received March 7, 1951.)

\section{1t. M. S. Robertson: A coefficient problem for functions regular in an annulus.}

Let $w=f(z)=\sum_{-\infty}^{\infty} a_{n} z^{n}$ be regular and single-valued in the annulus $0 \leqq \rho \leqq|z|<1$. For each $|z|=r$ in the range $\rho<r<1$ let the image of $|z|=r$ through the mapping $w=f(z)$ cross a given straight line $l$ precisely $2 p$ times ( $p$ fixed). Sharp bounds are obtained for the complex coefficients $a_{n},|n|>p$, in terms of $a_{k}, \pm k=0,1,2, \cdots, p$, and the parameters locating $l$. This is an extension of recent results by A. W. Goodman and M. S. Robertson [Trans. Amer. Math. Soc. vol. 70 (1951) pp. 127-136] where $a_{-n}=0$ for $n \geqq 0, a_{n}$ real for $n \geqq 1$, and where $l$ was the real axis. An application is made of the extension to prove that the Goodman conjecture for the coefficients of multivalent functions $F(z)=\sum_{1}^{\infty} a_{n} z^{n}$ of order $p$ in $|z|<1$ is correct at least for those $p$-valent functions $w=F(z)$ for which $\partial \Re F\left(r e^{i \theta}\right) / \partial \theta$ changes sign $2 p$ times on each circle $|z|=r, \rho<r<1$. (Received March 12, 1951.)

302. James Sanders: An existence theorem for some partial differential equations of the fourth order. Preliminary report.

Equations of the form (1) $L(u) \equiv u_{x x}+\left(\tau_{1}(y) \sigma_{2}(x) / \tau_{2}(y) \sigma_{1}(x)\right) u_{y y}+\left(\sigma_{1}^{\prime} / \sigma_{1}\right) u_{x}$ $+\left(\sigma_{2} \tau_{1} / \sigma_{1}\right)\left(1 / \tau_{2}\right)^{\prime} u_{y}=0$, a generalization of Laplace's equation, were considered by Bers and Gelbart (Trans. Amer. Math. Soc. vol. 56 (1944)). In this paper, equations of the form (2) $L L(\phi)=0$, generalization of the biharmonic equation, are considered. By an application of Green's theorem, the uniqueness of the solution of the boundaryvalue problem (3) $L L(\phi)=0, \phi=f(s), \partial \phi / \partial n=g(s)$, on $C$, is proved. $C$ is a simple, closed, analytic curve bounding the simply connected domain $D$. Existence of a solution of (3) is proved by first reducing (3) to a system of second order equations and then reducing the second order system to a system of integral equations. (Received March 8, 1951.)

\section{L. R. Sario: Alternating method for analytic functions.}

The alternating method for harmonic functions, created by Schwarz for surfaces with finite genus, can be generalized for arbitrary Riemann surfaces (C. R. Acad. Sci. Paris vol. 229 (1949) pp. 1293-1295; vol. 230 (1950) pp. 42-44 and 168-170). An analogous method for analytic functions can be developed and furnishes the following theorem. Consider on an arbitrary Riemann surface $R$ a finite set $\gamma$ of analytic Jordan curves, relatively bounding a domain $R_{1}$, compact or not. In the complement $R_{2}=R-R_{1}$ let $z$ be a complex function (in general multi-valued) which is analytic in a neighborhood of $\gamma$. If $\int_{\gamma} d z=0$, then there exists on $R$ a function w satisfying the fol- 
owing conditions: $1^{\circ} w$ is analytic and Dirichlet bounded in $R_{1}, 2^{\circ} w-z$ is analytic and Dirichlet bounded in $R_{2}$. The generalized alternating methods have applications to existence and mapping problems on Riemann surfaces (cf. problems in Annales Academiae Scientiarum Fennicae Ser. A. I. 50 (1948) pp. 1-79). In certain cases they allow a simpler approach than the Dirichlet principle or Hilbert space methods. (Received March 14, 1951.)

\section{4t. L. R. Sario: Linear operators on Riemann surfaces.}

On an arbitrary Riemann surface $R$ consider two compact domains $D$ and $F$ $(\bar{D} \subset F)$, bounded by finite sets $a$ and $b$ respectively of analytic Jordan curves. Let $u$ be a harmonic function in $\bar{F}-D$ such that $u=0$ on $a, \int_{a} d \bar{u}=0$, and denote by $v$ the harmonic function in $F$ with boundary values $u$ on $b$. The harmonic function in $R-D$ which minimizes the Dirichlet integral among functions with values $v$ on $a$ is a linear operator $L(u)$ of $u$. The sequence $\left\{h_{n}=\sum_{i=1}^{i=n} L^{i}(u)\right\}$ converges uniformly in $R-D$ towards a harmonic function $h$, bounded and Dirichlet bounded. The harmonic continuation of $u+h$ in $D$ is single-valued and regular. (Received March 14, 1951.)

\section{5t. Albert Schild: On a problem in conformal mapping of schlicht functions.}

Let $S^{*}$ be the class of functions $w=f(z)=z+\sum_{n=2}^{\infty} a_{n} z^{n}$, regular and schlicht in the unit circle, map the unit circle into a region $D^{*}$ in the $w$-plane, starshaped with respect to $w=0$. (The region $D^{*}$ is called starshaped with respect to $w=0$ if every point of $D^{*}$ can be joined to $w=0$ by a straight line segment lying entirely in $D^{*}$.) Let $d^{*}$ be the distance of the closest point of the boundary of $D^{*}$ from $w=0$, and let $d_{0}$ be the distance of the closest point of the map of $|z|=r_{0}$-where $r_{0}$ is the "Rundungs Schranke" (bound of convexity) of $w=f(z)$-from $w=0$. It has been conjectured that $d_{0} / d^{*} \geqq 2 / 3$ for all functions of the class $S^{*}$, the lower limit being attained by the function $w=f(z)=z(1+z)^{-2}$. The author has demonstrated the truth of this conjecture for certain subclasses of $S^{*}$, while for the general function of class $S^{*}$ certain lower bounds for $d_{0} / d^{*}$ have been obtained. The methods employed are of elementary nature. (Received February 21, 1951.)

306. E. W. Titt: Leibnitz rule for differentiating multiple integrals over arbitrary variable regions; extended to cases where the classical rule yields divergent integrals.

This paper presents rules for differentiating multiple integrals over arbitrary regions which make successive application of Leibnitz rule for single integrals unnecessary. By the new rules the derivative of an $n$-tuple integral becomes an $n$-tuple integral of the derived integrand plus an $(n-1)$-tuple integral (taken over the boundary of the region) of the original integrand multiplied by a determinant involving derivatives of the representation. The discovery of the rules was enhanced by a theory of multiple integration (by one of the authors) which inductively defines an $n$-tuple integral over an arbitrary region in terms of an $(n-1)$-tuple integral over the boundary. These rules have been extended so as to enable one to differentiate the integral of a scalar product where the classical rule yields divergent integrals. These latter rules perform three operations at one time: (1) an integration by parts, integrating one vector and differentiating the other; (2) the application of the aforementioned rule for differentiating multiple integrals; (3) reverse integrations by parts and simplifica- 
tion. The result is an easily applied rule involving $n,(n-1)$, and $(n-2)$-tuple integrals. (Received March 12, 1951.)

307. Alexander Weinstein: On the fundamental solutions of the Tricomi equation.

In a previous paper (A. Weinstein, Bull. Amer. Math. Soc. vol. 55 (1949) p. 520) two fundamental solutions of Tricomi's equation have been given in closed form for all values of the variables in the elliptic half-plane. It is shown now that both solutions can be extended to the hyperbolic half-plane where they are given in closed form by means of formulas derived from classical results on the equations of Euler-Poisson (G. Darboux, Leçons sur la thêorie gênêrale des surfaces, vol. 2, Paris, 1914, p. 68). (Received March 14, 1951.)

\section{8t. D. V. Widder: The Weierstrass transform of positive functions.}

Necessary and sufficient conditions are obtained in order that $f(x)$ should be of the form $\int_{-\infty}^{\infty} \exp \left[-(x-y)^{2} / 4\right] d \alpha(y)$, where $\alpha(y)$ is a nondecreasing function. They are: (1) $f(x)$ is a real entire function such that $f(x+i y)=O\left[\exp \left(-y^{2} / 4\right)\right]$ as $|y| \rightarrow \infty$, uniformly in every vertical strip; (2) $\exp \left(-t D^{2}\right) f(x) \geqq 0$ for $0<t<1,-\infty<x<\infty$. The symbolic operator (2) is interpreted as $(4 \pi t)^{-1 / 2} \int_{-\infty}^{\infty} \exp \left[-y^{2} / 4 t\right]\{\cos y D\} f(x) d y$, where $\{\cos y D\} f(x)$ is the obvious infinite series. The result is the analogue of the familiar Bernstein theorem concerning the representation of completely monotonic functions as Laplace transforms of positive functions. The chief tool is the author's result concerning positive temperatures on an infinite rod (Trans. Amer. Math. Soc. vol. 55 (1944)). (Received April 5, 1951.)

309t. Y. K. Wong: Some inequalities of determinants of Minkowski type.

Consider a matrix $a$ of order $n$ with non-negative elements. Let $M(a)$ be the supremum of $\left|x^{\prime} a y\right|$ for all $x, y$ on a unit Euclidean sphere. Let $D_{n}$ be the determinant of $I-a$ and $D_{n-1}$ the determinant of the minor $I-a_{n-1}$ which consists of the first $n-1$ rows and columns of $I-a$. If $M(a)<1$, then $D_{n}$ is bounded above by $[1-a(n, n)$ $\left.-\sum_{k=0}^{m} \sum_{i, j<n} a(n, i) a_{n-1}^{k}(i, j) a(j, n)\right] D_{n-1}$ for $m=1,2, \cdots$. The equality holds if $m=\infty$. If $M(a)<1$ is replaced by the condition that for every $j=1, \cdots, n, \sum_{i} a(i, j)$ $\leqq 1$ (summing for all $i \neq j$ ), $a(j, j)<1$, and $D_{n-1}$ is nonvanishing, the inequality holds for $m=1$. Under proper restriction, if a term $p \sum a(n, i) a_{n-1}^{m}(i, j) a(j, n) D_{n-1}$ is subtracted from the above expression, one gets a very good lower bound for $D_{n}$. The values for $p$ are determined under various conditions. (Received March 20,1951.)

\section{Applied Mathematics}

310t. G. S. S. Ludford: The behavior at infinity of the potential function of a two-dimensional subsonic compressible flow.

The well known expansion $w(z)=A z+B \log z+\sum_{n=1}^{\infty} C_{n} / z^{n}$ at infinity for the complex potential function of an incompressible fluid is transformed into the logarithmic plane, where it is seen to have a certain set of singularities at the image of the point $z=\infty$ (in the physical plane). By constructing a similar set of singularities in the pseudo-logarithmic plane, where the potential function of a compressible fluid satisfies a linear elliptic differential quation in normal form (S. Bergman, Trans. Amer. 
Math. Soc. vol. 62 (1947) p. 452), and then transforming back to the physical plane, the expansion $\phi=a r \cos \left(\theta-\theta_{0}\right)+b(\theta) \log r+c(\theta)+\sum_{m, n=0}^{\infty}\left(d_{m n}(\theta) / r^{n}\right)((\log r) / r)^{m}$ with $d_{00}$ constant is obtained, where $(r, \theta)$ are polar coordinates, and the series converges uniformly and absolutely for $r$ large enough. It is further shown that if $b(\theta), c(\theta) \equiv 0$, that is, there is no source or circulation in the flow, then $d_{m n}(\theta) \equiv 0$ for $m \neq 0$, so that the logarithmic terms disappear. On the basis of these expansions the force and moment formulae for an incompressible flow may be extended to subsonic compressible flow. The reference above gives an alternative derivation of these formulae. (Received March 12, 1951.)

\section{1t. L. A. Zadeh: Theory of filtering of signals in a $\lambda$-domain.}

An integral representation of a signal $u(t)$ in the form $u(t)=\int_{C} k(t ; \lambda) U(\lambda) d \lambda$ may be interpreted as the resolution of $u(t)$ in terms of $k(t ; \lambda)$, with $U(\lambda)$ playing the role of a weighting function. $U(\lambda)$ is related to $u(t)$ by $U(\lambda)=\int_{-\infty}^{\infty} k^{-1}(t ; \lambda) u(t) d t$ where $k^{-1}(\lambda ; t)$ is the inverse of $k(t ; \lambda)$. Let $u(t)$ be the input to a linear system $N$ and let $v(t)$ be the response of $N$ to $u(t) . v(t)$ is given by $v(t)=\int_{C} K(t ; \lambda) U(\lambda) d \lambda$ where $K(t ; \lambda)$ is the response of $N$ to $k(t ; \lambda)$ and is called a characteristic function of $N$. For the particular case where $k(t ; \lambda)$ is a delta function $\delta(t-\xi), \lambda=\xi$, the characteristic function becomes the impulsive response $W(t, \xi)$ or Green's function of $N . W(t, \xi)$ is related to $K(t ; \lambda)$ by $W(t, \xi)=\int_{C} K(t ; \lambda) k^{-1}(\xi ; \lambda) d \lambda$. A system $N$ is said to be an ideal filter if $K(t ; \lambda)=k(t-\beta ; \lambda)(\beta=\mathrm{a}$ constant) for $\lambda$ in a region $R$ of the $\lambda$-plane, and $K(t ; \lambda)=0$ for $\lambda$ not in $R$. Two signals $u_{1}(t)$ and $u_{2}(t)$ are called separable if in some $\lambda$-domain (that is, for some $k(t ; \lambda)) U_{1}(\lambda)=0$ for $\lambda$ in $R$ and $U_{2}(\lambda)=0$ for $\lambda$ not in $R$. Such signals can be physically separated from each other by using an ideal filter in this domain. (Received March 9, 1951.)

\section{Geometry}

\section{H. S. M. Coxeter: Interlocked rings of spheres.}

Dupin's cyclide is the common envelope of two systems of spheres [A. Mannheim, Nouvelles Annales de Mathematiques vol. 19 (1860) p. 71]. Any sphere of either system belongs to a sequence of spheres in that system, each touching the next (and all touching every sphere of the other system). By means of an excursion into fourdimensional space it is proved that, if one system contains a closed sequence (the last sphere touching the first), then the other system likewise contains a closed sequence. The figure consists of a cycle of $n$ spheres interlocked with a cycle of $m$, where either $m=2 n$ with $n$ odd, or $2 m=n$ with $m$ odd, or $m=n \equiv 0(\bmod 4)$. The case when $m$ and $n$ are 3 and 6 was noticed by F. Soddy [Nature vol. 138 (1936) p. 958]. (Received March 14, 1951.)

\section{P. C. Hammer and Andrew Sobczyk: Planar line families. I.}

Let $F$ be a family of lines in the plane which simply covers all points, including points at infinity, exterior to some circle. Then there exists a convex body $C$ the essential diameters of which extend to give precisely all lines in $F$. Moreover, $C$ may be taken as a body with constant diameter length and with a smooth boundary curve. It is demonstrated how all planar convex bodies of constant diameter length may be constructed given all such families of lines. Given all continuous increasing functions mapping the closed interval $[0,1]$ biuniquely into $[0,1]$, all such families of lines are constructed. (Received February 14, 1951.) 


\section{4t. A. J. Hoffman: On chains.}

Let $E$ be a field admitting an involutoric automorphism having $F$ as fixed field, let $m$ be the projective line over $E$ and $n \subset m$ the projective line over $F$. Define a chain in $m$ as the image of $n$ under any projective transformation of $m$. In the classical case, $E$ is the complex numbers, and the chains can be regarded as the circles of the real inversion plane. C. T. Yang (Duke Math J. (1948)) has shown that many of the properties of chains in the classical case hold also if $E$ is $G F\left(p^{2^{k}}\right)$. The purpose of this note is to point out that these properties hold in the general case. In fact, stronger results, such as Miquel's theorem, are valid in general. (Received March 13, 1951.)

315t. Ira Rosenbaum: A new system of completely independent postulates for betweenness.

Huntington and Kline (Trans. Amer. Math. Soc. (1917)) considered 12 postulates for betweenness, from which 11 sets of independent postulates were selected. Eight of the eleven sets of independent postulates contained 6 postulates each, three contained 7 each. Huntington (Trans. Amer. Math. Soc. (1924)) obtained a set of 5 completely independent postulates for betweenness by adding to the original 12 postulates a 13th. The present paper indicates that a set of 5 completely independent postulates for betweenness may be selected from Huntington and Kline's original set of twelve, by slightly modifying two of the postulates, without bringing in any essentially novel postulates as Huntington did. The postulates $A, B, D$ of Huntington and Kline's paper are retained; their postulates 1 and 2 are modified by removing the hypothesis that $A, B, X, Y$ are distinct, and thereafter cited as $1^{\prime}$ and $2^{\prime}$. Mr. Walter E. Roop, a student of the author, noted that from $A, B, D$ and the author's $1^{\prime}$ and $2^{\prime}, \sim(A X Y$ . $A Y X)$ is derivable. It follows easily that Huntington and Kline's postulate $C$ is derivable; moreover $1^{\prime}$ implies 1 , and $2^{\prime}$ implies 2 . Hence $A, B, D, 1^{\prime}$, and $2^{\prime}$ imply $A, B, C, D, 1$, and 2 (Huntington and Kline's first set of 6 independent postulates for betweenness). Interpretations showing the postulates $A, B, D, 1^{\prime}$, and $2^{\prime}$ to be completely independent, in the sense of E. H. Moore, are given. (Received January 18, 1951.)

\section{Statistics AND Probability}

\section{K. L. Chung and Jacob Wolfowitz: On a limit theorem in re- newal theory.}

Let $X_{1}, X_{2}, \cdots$ be independent, identically distributed, integral-valued chance variables. Let $d$ be the absolute value of the greatest common divisor of the values which each variable can assume with positive probability. Let $m=E\left(X_{n}\right)$ be the mathematical expectation of each $X_{n}$ and let $S_{n}=\sum_{1}^{n} X_{k}$. Suppose $0<m \leqq \infty$. The authors prove that $\lim \sum_{n=1}^{n=\infty} P\left(S_{n}=x d\right)=d / m$ or 0 , according as $x$ approaches $+\infty$ or $-\infty$ through integral values. In the case where the $X_{n}$ 's assume only non-negative integral values this was proved by Kolmogorov and Erdös, Feller, and Pollard. (Received February 26, 1951.)

\section{L. A. Zadeh: On stochastic Heaviside operators.}

The result of operation with a time-dependent Heaviside operator $H(p ; t)$ on $u(t)$ is given by $H(p ; t) u(t)=(1 / 2 \pi) \int_{-\infty}^{\infty} H(j \omega ; t) U(j \omega) e^{j \omega t} d \omega$ where $U(j \omega)$ is the Fourier transform of $u(t)$. An $H(p ; t)$ which is a random function of $t$ involving $p$ as a fixed parameter is called a stochastic Heaviside operator. Let $u(t)$ and $H(p ; t)$ be independ- 
ent and stationary in the wide sense, and let $v(t)=H(p ; t) u(t)$. Furthermore, let $\psi_{u}(\tau)=\overline{u(t) u(t+\tau)}, \psi_{v}(\tau)=\overline{v(t) v(t+\tau)}$, and $\psi_{H}(p ; \tau)=\overline{H(p ; t) H(-p ; t+\tau)}$ be the correlation functions of $u(t), v(t)$, and $H(p ; t)$. It can readily be shown (Bull. Amer. Math. Soc. Abstract 56-4-329) that these correlation functions are related to each other by the operational relation $\psi_{v}(\tau)=\psi_{H}(p ; \tau) \psi_{u}(\tau)$. Consider two independent stochastic operators $H_{1}$ and $H_{2}$ and let $H_{3}$ be their product $H_{3}=H_{1} * H_{1}$ (the product of $H_{1}$ and $H_{2}$ is given by the operational relation $H_{3}(s ; t)=H_{1}(p+s ; t) H_{2}(s ; t)$ where $s$ is a parameter). Let $\psi_{1}, \psi_{2}$, and $\psi_{3}$ be the correlation functions of $H_{1}, H_{2}$, and $H_{3}$. It is shown that $\psi_{3}=\psi_{1} * \psi_{2}$. This result is useful in the determination of the correlation functions of complex waveforms. (Received March 13, 1951.)

\section{TOPOLOGY}

\section{Raoul Bott: Nondegenerate critical manifolds. Preliminary} report.

Let $M$ be a real $n$-dimensional compact manifold with a three times differentiable structure. Let $f$ be a real-valued function of a class $C$ on $M$. A submanifold $N$ of $M$ is said to be a nondegenerate critical manifold of $f$ if $(1) \partial f / \partial x^{i}=0(i=1, \cdots, n)$. (2) $\operatorname{rank}\left(\partial^{2} f / \partial x^{i} \partial x^{i}\right)=\operatorname{dim} M-\operatorname{dim} N$, on $N$. It is shown that the Morse type numbers $M$ of this critical set are given by $M_{N}^{i}=R^{i-\lambda}(N)$, where $\lambda$ is the number of negative characteristic values of $\left(\partial^{2} f / \partial x^{i} \partial x^{i}\right)$ on $N$, and $R^{s}(N)$ is the $s$-dimensional Betti number $\bmod 2$ of $N$. When $N$ is a point $P$ this reduces to the early result of Morse: $M_{P}^{i}=\delta_{\lambda}^{i}$. Applications of this lemma are made to fibre bundles and a generalized form of the billiard ball problem. (Received January 15, 1951.)

\section{9t. Felix Browder: On the fixed-point index. I.}

If $X$ is a compact neighborhood retract of a Tychonoff cube, a fixed-point index, a non-negative integer $i(O, f)$, is defined for all continuous mappings of $\bar{O}$ into $X$ where $O$ is open in $X$ and $f$ has no fixed-points on the boundary of $O$. This index is shown to have the basic properties of the classical fixed-point index defined for selfmappings of finite polytopes and is a proper generalization of the latter as well as coinciding with the Leray index for any neighborhood retract of a Tychonoff cube which is convexoid. By a simple extension to the case in which only the image under $f$ needs to be compact, the definition of this paper becomes a proper generalization of the Schauder-Leray index obtained from the degree. The definition is carried through by the use of approximating mappings of the nerves of finite open coverings of $X$ which are obtained from the given mapping by applying the Alexandroff mapping theorem and homotopy local connectedness properties of $X$. A generalization is given also of the Nielsen Reidemeister-Wecken theory of fixed-point classes. (Received March 15, 1951.)

\section{0t. Felix Browder: On the fixed-point index. II.}

If $X$ is a compact, not necessarily metric, HLC* space, a fixed-point index $i(O, f)$ is defined for all continuous mappings of $\bar{O}$ into $X$ where $O$ is an open subset of $X$ and $f$ has no fixed points on the boundary of $O$. The index is defined by the use of a chain derivation similar to, but not identical with, that defined for quasi-complexes in Lefschetz' Algebraic topology. Using linear endomorphisms, induced by the given mapping, of the rational chain groups of subcomplexes of the nerves of finite open coverings of $X$, the definition and proof of the properties of the fixed-point index 
make no use of properties of the classical fixed-point index for finite polytopes. The index as defined for compact HLC* spaces constitutes a proper generalization of all previous definitions of the fixed-point index. (Received March 15, 1951.)

\section{Mary E. Estill: Concerning separability.}

Let axiom 1.3 denote the axiom resulting from the omission of condition 4 in the statement of R. L. Moore's Foundations of point set theory. In this paper there is shown the existence of a connected and connected im kleinen space satisfying axiom 1.3 which is not separable and which does not contain uncountably many mutually exclusive domains. There is also shown the existence of a connected space satisfying axiom 1.3 which is not separable and which does not contain uncountably many mutually exclusive domains such that, if $A$ is a point of a region $R$ and $B$ is a point distinct from $A$, there exists a point lying in $R$ separating $A$ from $B$. However, there does not exist a connected im kleinen space satisfying axiom 1.3 which is not separable and does not contain uncountably many mutually exclusive domains such that, if $A$ is a point of a region $R$ and $B$ is a point distinct from $A$, there exists either a separable point set lying in $R$ separating $A$ from $B$ or a finite point set separating $A$ from $B$. (Received March 15, 1951.)

\section{2t. W. S. Massey: Applications of exact couples in algebraic topol-} ogy.

Exact couples of groups have many applications to algebraic topology, of which three will be mentioned here. The first application is obtained by considering relative and absolute homotopy groups of the various skeletons of a cell complex. The exact couple thus obtained is not a topological invariant of the complex, but the successive derived couples are invariants. The first derived couple contains among other things an exact sequence recently considered by J. H. C. Whitehead [Ann. of Math. vol. 52 (1950) pp. 51-110]. The second application is obtained by considering the SpanierBorsuk cohomotopy groups of the various skeletons of a cell complex. The first derived couple is again a topological invariant, and it contains the cohomotopy groups of the complex, and various cohomology groups. The third application is to the problem of obtaining relations between the homology groups of the base space, total space, and fiber in a fiber bundle. The geometrical basis for this application is to be found in recent work of Spanier and Chern [Proc. Nat. Acad. Sci. U.S.A. vol. 36 (1950) pp. 248-255]. (Received March 15, 1951.)

\section{3t. W. S. Massey: The algebra of exact couples of groups.}

An exact couple of groups consists of two abelian groups, $A$ and $C$, homomorphisms $g: A \rightarrow C$ and $h: C \rightarrow A$, and an endomorphism $f: A \rightarrow A$. These groups and homomorphisms are required to satisfy the following "exactness" conditions: image $f=$ kernel $g$, image $g=$ kernel $h$, and image $h=$ kernel $f$. Define an endomorphism $d: C \rightarrow C$ by $d=g h$. Then $d$ is a differential operator, that is, $d^{2}=0$. Let $C^{\prime}$ denote the derived group of $C$ with respect to this differential operator and let $A^{\prime}=f(A)$. Then $f$ defines an endomorphism $f^{\prime}: A^{\prime} \rightarrow A^{\prime}, h$ defines a homomorphism $h^{\prime}: C^{\prime} \rightarrow A^{\prime}$, and $g f^{-1}$ defines a homomorphism $g^{\prime}: A^{\prime} \rightarrow C^{\prime}$. The groups $A^{\prime}$ and $C^{\prime}$ together with the homomorphisms $f^{\prime}, g^{\prime}, h^{\prime}$ satisfy the exactness condition mentioned above, and hence constitute an exact couple, called the first derived exact couple. This process can be repeated to obtain the second derived couple, and so on. In applications to topology, the groups $A$ and $C$ usually also have a bi-graded structure, and the homomorphisms 
$f, g$, and $h$ are homogeneous. In an analogous manner, one can consider exact couples of vector spaces, modules, rings, or algebras. (Received March 15, 1951.)

\title{
324t. Philip Rabinowitz: Normal coverings and uniform spaces.
}

The structure of uniform spaces is investigated using graphs of normal coverings. The following two theorems are the main results of this investigation: (1) The collection of graphs of all locally-finite normal coverings of a uniformizable space $X$ forms a base of the universal uniformity of $X$. (2) Every fully normal space is complete relative to its universal uniformity. Since Stone (Bull. Amer. Math. Soc. vol. 54 (1948)) has proven the equivalence of paracompactness and full normality for Hausdorff spaces, the latter theorem answers in the affirmative the problem posed by Dieudonné (J. Math. Pures Appl. vol. 23 (1944)). (Received February 16, 1951.)

\author{
L. W. Cohen, \\ Associate Secretary
}

